\title{
Simple Linearized Equation of Motion for Spinning Tops on a Table*
}

\author{
Chikara MURAKAMI ${ }^{* *}$ \\ **The Japan Society of Mechanical Engineers, \\ Shinanomachi-RengakanBldg., Shinanomachi 35, Shinjuku-ku, Tokyo, 160-0016 Japan \\ E-mail: 45189901@d1.dion.ne.jp
}

\begin{abstract}
This paper deals with deriving a simple linearized equation of motion for axisymmetric tops spinning on a table; the equation includes the effects of both friction and gravitation. The derived equation is a single second-order equation with complex number coefficients. The main variable is the inclination angle of the spinning axis, which is expressed by a single complex number. No Euler's angle is used. The precondition for linearization is that both the angular momentum vector and the spinning axis are close to the zenith direction. The friction is assumed to be viscous, and the friction torque can be expressed by using the height of the gravity center and the radius of the curvature at the contact point to the table. Two eigenvalues for the equation give a deep insight into the attitude behavior. The root-locus method was applied to explain the spinning motion stability of various axisymmetric bodies. An analytical solution for a horizontally suspended spinning top is given to show that the locus of the spinning axis is a trochoid.
\end{abstract}

Key words: Stability, Nutation, Precession, Gyro, Spinning Top, Attitude Motion, Magnetic Bearing, Root-locus Method

\section{Introduction}

In 1995, the author derived an imperfect simple second-order attitude equation of motion for spinning tops on a table, including two external torques: gravitational and frictional. ${ }^{(1)}$ However, the external torques of this equation were not given in concrete terms at that time. In this paper, they are expressed with concrete dimensions.

The torque-free conical motion of an axisymmetric spinning rigid body around the angular momentum vector $\boldsymbol{H}$ is known as free precession and was clarified geometrically more than a century ago. The body cone rolls around the fixed space cone whose center axis is $\boldsymbol{H}$, and the spinning axis $\boldsymbol{S}$ draws a cone. The most important vector, which is the rotating velocity of $\boldsymbol{S}$ around $\boldsymbol{H}$-shown as $\boldsymbol{\Omega}$ in this paper-has been derived accurately. Nowadays, this free coning motion is called nutation in space engineering; later, this nomination will be shown to be very reasonable. This coning motion or nutation should be considered as the basis for the rotating motion. In this paper, the coning motion observed from inertial space is expressed as a circular motion drawn by the end of the spinning unit-length axis on a fixed inertial complex plane, which has a real and imaginary axis. Euler's angle is not needed to express the circular motion by using this complex coordinate system. Therefore, the equation of motion of the title can be derived provided that both $\boldsymbol{H}$ and $\boldsymbol{S}$ have small inclination angles to the vertical direction. From the equation, two eigenvalues can be easily derived; therefore, we can then describe root-locus diagrams, which give us an intuitive understanding of the stability affected by the shapes of tops and other axisymmetric [DOI: 10.1299/jsdd.4.142]

Copyright () 2010 by JSME 
spinning bodies. An analytical solution and its mechanical model for a horizontally suspended spinning top are given and show that the locus of the spinning axis is a trochoid. These results are extremely effective for not only intuitive understanding of the tops but also for designing a control system of magnetic bearings. In this study, translational motion was neglected.

\section{Nomenclature and Coordinate Systems}

In this study, three cubic coordinate systems (inertial-, body-, and despin-) assuming the center of gravity $G$ as the origin were used, as shown in Fig. 1 . The inertial $Z$-axis is the vertical axis. The body coordinates axes are the three principal axes $x, y$, and $z$, where the $z$-axis is the axis of symmetry and called the spin-axis in this paper. Let $A, A, C$, be the moment of inertia (MOI) about the $x, y, z$ axes, respectively. In subsequent sections, "polar" denotes the $z$ direction, and "radial" denotes the direction perpendicular to the polar one. The subscripts $p$ and $r$ express components of these directions. The ratio (polar MOI)/(radial $\mathrm{MOI})=C / A=\sigma$ is called the MOI ratio.

The three-dimensional vectors are expressed in Gothic fonts. If they are expressed in a row matrix form, the subscripts I, B, and D of the matrix denote the coordinate system of the components (I: inertial, B: body, and D: despin, respectively). The superscript $\mathrm{T}$ of the matrix denotes transposition of the matrix. For example, the main attitude vectors in row matrix form are as follows:

angular velocity of the body $\omega=\left[\omega_{x} \omega_{y} n\right]_{\mathrm{D}}^{\mathrm{T}}$

angular velocity of the despin coordinate system $\omega_{d}=\left[\omega_{x} \omega_{y} 0\right]_{\mathrm{D}}^{\mathrm{T}}$

angular momentum vector of the body $\boldsymbol{H}=\left[A \omega_{x} A \omega_{y} C n\right]_{\mathrm{D}}^{\mathrm{T}}$

unit vector of the spin axis (small inclination) $\boldsymbol{S} \equiv\left[\begin{array}{lll}0 & 0 & 1\end{array}\right]_{\mathrm{D}}^{\mathrm{T}} \approx\left[\begin{array}{lll}X & Y & 1\end{array}\right]_{\mathrm{I}}^{\mathrm{T}}$

where " $\equiv$ " means definition, and $X, Y<<1$ in Eq. (2.4).

The despin coordinate system is used in space engineering when the attitude inclination is small, and the spin velocity $n$ in Eq. (2.1) is zero, as shown in Eq. (2.2).

The direction of the spin axis $\boldsymbol{S}$ can be expressed as a point on the inertial unit sphere whose center coincides with the mass center $G$. If the inclination angle $\theta$ of $\boldsymbol{S}$ to the $Z$-axis is small, then the region occupied by $\boldsymbol{S}$ is restricted to a small one, which can be approximated by a horizontal plane with an inertial complex coordinate system with axes parallel to the two inertial horizontal axes, as shown in Fig. 1. Complex vectors on this plane are expressed by underlined characters. In this case, $\cos \theta \approx 1$ and $\sin \theta \approx \theta$; therefore, Eq. (2.4) can be converted to a complex vector $\underline{S}$ using the approximated coordinate transformation matrix, provided that $\theta_{X}$ and $\theta_{Y}$ are small inclination angles of $S$ around the $X$-and $Y$-axes, respectively:

$$
\begin{aligned}
& \quad\left[\begin{array}{ccc}
1 & 0 & 0 \\
0 & 1 & -\theta_{X} \\
0 & \theta_{X} & 1
\end{array}\right]\left[\begin{array}{ccc}
1 & 0 & \theta_{Y} \\
0 & 1 & 0 \\
-\theta_{Y} & 0 & 1
\end{array}\right]\left[\begin{array}{l}
0 \\
0 \\
1
\end{array}\right]_{\mathrm{D}}=\left[\begin{array}{ccc}
1 & 0 & \theta_{Y} \\
\theta_{X} \theta_{Y} & 1 & -\theta_{X} \\
-\theta_{Y} & \theta_{X} & 1
\end{array}\right]\left[\begin{array}{l}
0 \\
0 \\
1
\end{array}\right]_{\mathrm{D}}=\left[\begin{array}{c}
\theta_{Y} \\
-\theta_{X} \\
1
\end{array}\right] \approx\left[\begin{array}{c}
X \\
Y \\
1
\end{array}\right]_{\mathrm{I}} \\
& \quad \therefore \quad \underline{S} \equiv X+\mathbf{i} Y \approx \theta_{Y}-\mathbf{i} \theta_{X} \equiv-\mathbf{i} \underline{\theta} \\
& \text { where } \mathbf{i} \equiv \sqrt{-1}, \text { and } \\
& \quad \underline{\theta} \equiv \theta_{X}+\mathbf{i} \theta_{Y}=\mathbf{i} \underline{S} .
\end{aligned}
$$

Although the real axis is parallel to the $X$-axis, the scale is $\theta_{Y}$; and though the imaginary axis is parallel to the $Y$-axis, the scale is $-\theta_{X}$, as shown in Fig.1.

The two radial velocities $\omega_{x}$ and $\omega_{y}$ are nearly horizontal and their directions are near to the $X$ - and $Y$-directions, respectively. Accordingly,

$$
\omega_{x} \approx \dot{\theta}_{X}, \omega_{y} \approx \dot{\theta}_{Y} \quad \text { (dot marks mean the time derivative of variables). }
$$

Using the complex number notation, their resultant velocity $\omega_{r}$ can be expressed as

$$
\underline{\omega}_{r} \equiv \omega_{x}+\mathbf{i} \omega_{y} \approx \underline{\dot{\theta}} \equiv \dot{\theta}_{X}+\mathbf{i} \dot{\theta}_{Y} .
$$

The initial values of time-dependent variables are shown by the subscript zero, for example, 


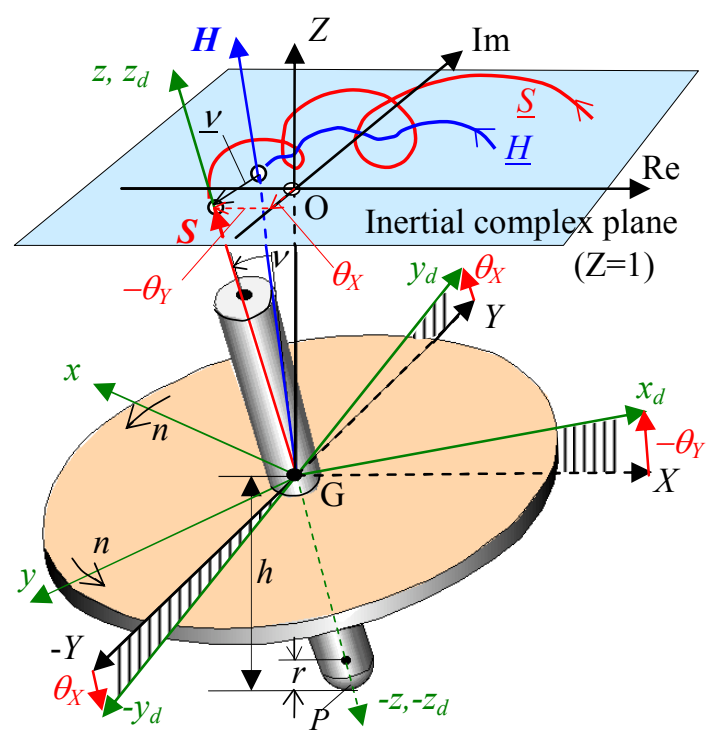

Coordinate systems : Inertial : G-XYZ Body : G- $x y z$ Despin : G- $x_{d} y_{d} z_{d}$ Inertial complex : O-ReIm Attitude vectors :

$\boldsymbol{H}$ : Angular momentum $S:$ Spin axis (unit length) Attitude angles of $S$ :

$\theta_{\mathrm{X}}:$ around $\mathrm{X}$-axis $\theta_{\mathrm{Y}}:$ around $\mathrm{Y}$-axis $v:$ from $\boldsymbol{H}$ to $\boldsymbol{S}$

Complex vectors :

Underlined characters $\underline{S}=\theta_{\mathrm{Y}}-\mathbf{i} \theta_{\mathrm{X}} \equiv-\mathbf{i} \underline{\theta}$ $\underline{H}=\underline{S}-\underline{v}$

$\mathrm{G}:$ Mass center, height $=h$

$P$ : Contact point to table, Radius of curvature $=r$

Fig. 1 Coordinate Systems and Main Nomenclatures

$$
\underline{H}_{0} \equiv \underline{H}(\mathrm{t}=0)
$$

As shown in Fig.1, $v$ and $\underline{v}$ are the half-cone angle and distance measured from $\underline{H}$ to $\underline{S}$, respectively. The contact point $P$ is on a spherical surface of curvature radius $r$ whose center is on the $z$-axis, and its distance from $\mathrm{G}$ is $h-r . Q$ and $q$ are external torques.

\section{Complex Number Expression of Attitude Vectors and External Torques}

As shown in the right part of Fig. 2, it is well known that $\omega$ can be decomposed to the $\boldsymbol{H}$ and $\boldsymbol{S}$ directions. The former is denoted by $\Omega$ and is the nutation velocity observed from inertia space, whereas the latter $\boldsymbol{n}_{s}$ is the nutation velocity observed from the rotating body. The well-known relation between $\boldsymbol{H}$ and $\boldsymbol{\Omega}$ is

$$
\boldsymbol{H}=A \boldsymbol{\Omega}=\boldsymbol{H}_{p}+\boldsymbol{H}_{r}
$$

In case of a small inclination,

$$
\begin{aligned}
& H_{p}=C n \approx A \Omega, \\
& \Omega \approx \sigma n, \\
& n_{s} \approx n-\Omega \approx(1-\sigma) n \\
& \underline{H}_{r}=A \underline{\omega}_{r} \approx A \dot{\dot{\theta}} \\
& v \equiv-\tan ^{-1}\left[H_{r} / H_{p}\right] \approx-H_{r} / H_{p} \\
& \underline{v} \approx-\underline{H} \underline{H}_{r} / H_{p} \approx-\underline{\dot{\theta}} / \Omega \quad \text { (distance from } \underline{H} \text { to } \underline{S} \text { ) } \\
& \underline{H}=\underline{\underline{S}}-\underline{v} \quad \text { or } \underline{H}=\underline{\dot{\theta}} / \Omega-\mathrm{i} \underline{\theta}
\end{aligned}
$$

To obtain a simple equation of motion, viscous-type friction torque - which is relatively small and proportional to the slipping velocity of the contact point $P$-was adopted. Figure 2 shows the relations between the attitude vectors and $P$ using a new complex coordinate plane, which agrees with the table plane and lies under $h$ from G. The new complex plane is $\mathrm{Z}=-h$ and distances on this plane have the dimension of length. The three attitude vectors $\boldsymbol{H}$, $S$, and $\omega$ are included in a plane; therefore, their positions (multiplied by $-h$ ) on this coordinate plane are on the straight line. On the other hand, the position of $P$ on this plane $h \underline{P}$ is on a line connecting $-h \underline{S}$ and the origin $\mathrm{Z}$ because of shape symmetry. Therefore,

$$
h \underline{P}=-(h-r) \underline{S}=\mathbf{i}(h-r) \underline{\theta}
$$

The vector $\omega$ is an instantaneous rotation axis of the body, and the distance from - $h \underline{\omega}$ to $h \underline{P}$ is its gyration radius $\varrho$ from which the velocity of $P$, or $\underline{V}$, is easily derived. From Fig.2,

$$
\begin{aligned}
& \underline{\omega}=\underline{S}+\underline{\dot{\theta}} / n \\
& \underline{\rho}=h \underline{P}-(-h \underline{\omega}) \approx r \underline{S}+h \underline{\dot{\theta}} / n
\end{aligned}
$$



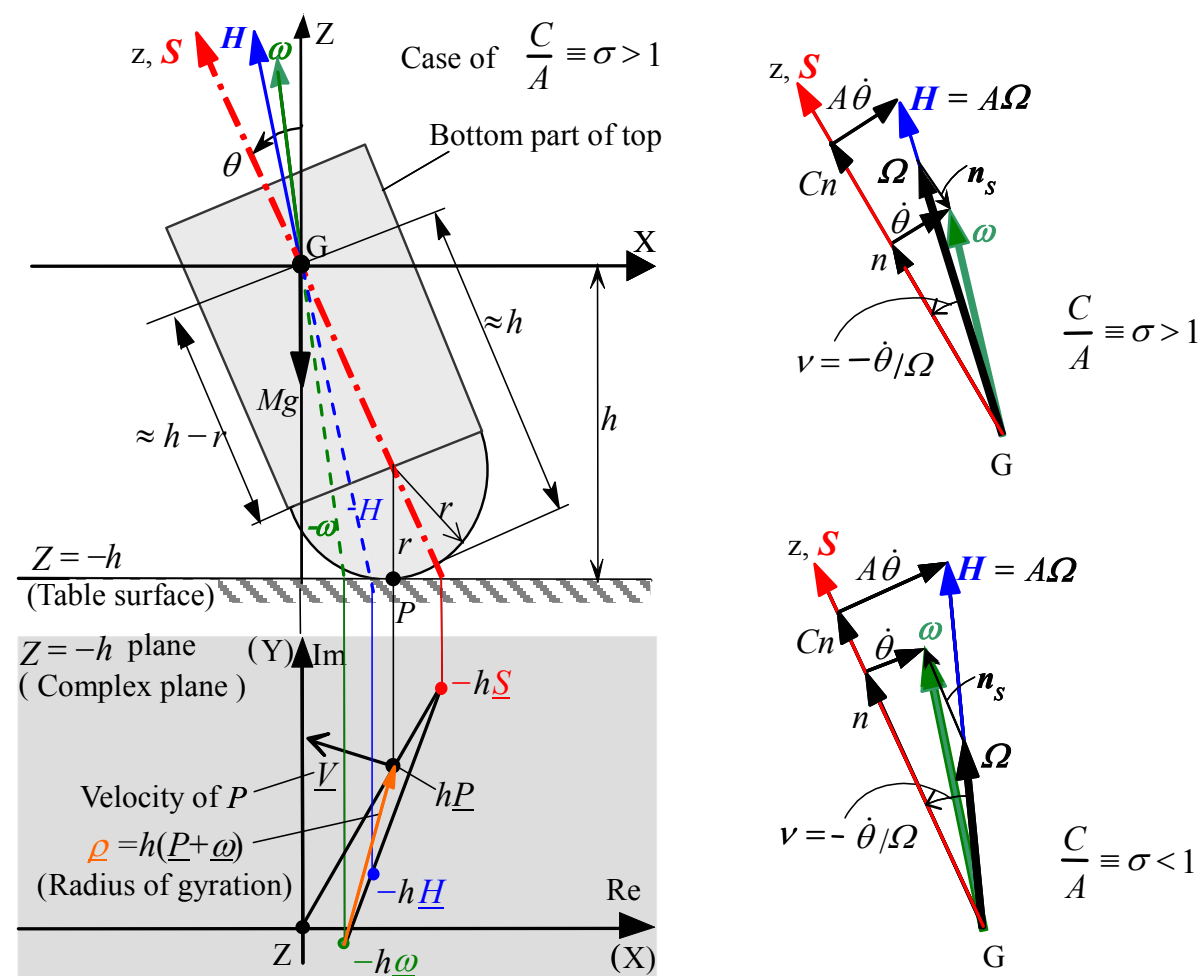

Fig. 2 Explanation of attitude vectors and contact point $\mathrm{P}$.

$$
\underline{V}=\mathbf{i} n \underline{\rho}=n r \underline{\theta}+\mathbf{i} h \underline{\dot{\theta}} .
$$

The friction force $\underline{F}$ is $-\mu \underline{V}$ where $\mu$ is a friction coefficient; therefore,

$$
\underline{F}=-\mu \underline{V}=-\mathbf{i} \mu(-\mathbf{i} n r \underline{\theta}+h \underline{\dot{\theta}}) \text {. }
$$

The main part of the friction torque $\boldsymbol{Q}_{\mathrm{f}}$ is proportional to $-\boldsymbol{h} \times \boldsymbol{F}$, whose direction is almost horizontal and whose phase angle is $-\pi / 2$ to $\underline{F}$.

$$
Q_{\mathrm{f}}=-\mathbf{i} h \underline{F}=-\mu h(-\mathbf{i} h r \underline{\theta}+h \underline{\dot{\theta}})=-\mu h\{-\mathbf{i}(\Omega / \sigma) r \underline{\theta}+h \underline{\dot{\theta}}\} \equiv-K_{N} \underline{\dot{\theta}}+\mathbf{i} \Omega K_{P} \underline{\theta}
$$
where

$K_{N} \equiv \mu h^{2}$ : damping coefficient,

$K_{P} \equiv \mu h r / \sigma:$ effective spring coefficient.

The rest is denoted as $Q_{Z}$ and is the deceleration torque; it is obtained from Eqs. (3.9), (3.13) and (3.7):

$\left|Q_{Z}\right| \leq|h \underline{P}||\underline{F}| \approx|h-r||\underline{\theta}(\mu n)| \mathbf{i} r \underline{\theta}+\sigma h \underline{y} \mid=$ second order of small quantities. Another external torque is the gravitational torque $\boldsymbol{Q}_{\mathrm{g}}$, which is a vector product of the vertical gravity force $M g$ and horizontal vector $h \underline{P}$; it has only a horizontal component whose phase angle to $\underline{P}$ is $-\pi / 2$. Using Eq. (3.9) for $h \underline{P}$,

$$
\begin{aligned}
Q_{\mathrm{g}} & =-\mathbf{i} M g h \underline{P}=M g(h-r) \underline{\theta} \equiv K_{g} \underline{\theta}=\mathbf{i} K_{g} \underline{S} . \\
K_{g} & \equiv M g(h-r): \text { gravity torque coefficient. }
\end{aligned}
$$

As shown above, the two main external torques $\boldsymbol{Q}_{\mathrm{f}}+\boldsymbol{Q}_{\mathrm{g}}$ can be concisely expressed using the attitude information.

\section{Derivation of a Linearized Equation of Motion for Spinning Tops.}

First, Euler's moment equation is used, where the moving coordinate is the despin one. All components of all vectors are expressed by the inertial coordinates.

$$
\frac{d \boldsymbol{H}}{d t}=\frac{\partial \boldsymbol{H}}{\partial t}+\omega_{d} \times \boldsymbol{H}=\boldsymbol{Q} \equiv \boldsymbol{Q}_{\mathrm{f}}+\boldsymbol{Q}_{\mathrm{g}}
$$




$$
\left.\begin{array}{c}
A \ddot{\theta}_{X}+C n \dot{\theta}_{Y}=Q_{X} \\
A \ddot{\theta}_{Y}-C n \dot{\theta}_{X}=Q_{Y} \\
C \dot{n}=Q_{Z}
\end{array}\right\}
$$

Assuming a small inclination $Q_{Z} \approx 0$ from Eq. (3.17), then, from Eq. (3.2)

$$
C n \approx \text { const. }=A \Omega
$$

In Eq. (4.2), by multiplying $\mathbf{i}$ to the 2 nd line equation and adding it to the 1st line, complex notation is used to obtain the following time invariant coefficient equation:

$$
A \underline{\ddot{\theta}}-\mathbf{i} A \Omega \underline{\dot{\theta}}=\underline{Q}=\underline{Q}_{f}+\underline{Q}_{g}=-K_{N} \underline{\dot{\theta}}+\mathbf{i} \Omega K_{P} \underline{\theta}+K_{g} \underline{\theta} .
$$

This equation is rearranged to

$$
A \underline{\ddot{\theta}}+\left(K_{N}-\mathbf{i} A \Omega\right) \underline{\dot{\theta}}-\left(\mathbf{i} \Omega K_{P}+K_{g}\right) \underline{\theta}=0 .
$$

After dividing the above equation by $A$ and changing notations to $K_{N} / A \equiv k_{N}, K_{P} / A \equiv k_{P}$, and $K_{g} / A \equiv k_{g}$, the following is obtained:

$$
\underline{\ddot{\theta}}+\left(k_{N}-\mathbf{i} \Omega\right) \underline{\dot{\theta}}-\left(\mathbf{i} \Omega k_{P}+k_{g}\right) \underline{\theta}=0 .
$$

Equation (4.5) or (4.6) is the fundamental linearized equation for spinning tops.

\section{Another Method for Deriving the Equation.}

Nutation can be considered as a circular motion drawn by the head of the unit vector $\boldsymbol{S}$. The center of the circle is vector $\boldsymbol{H}$, which coincides with the center axis for the cone of the spin axis. When both $\boldsymbol{H}$ and $\boldsymbol{S}$ are close to the $Z$-axis or vertical direction, the surface of the circle is nearly horizontal. Therefore, this circular motion can be described by the same complex variables used in the previous section.

The moving radius $\underline{u}(\mathrm{t})$ with rotation velocity $\Omega$ can be expressed as follows:

$$
\underline{v}(t)=\underline{v}_{0} \mathrm{e}^{\mathrm{i} \Omega t} \text {. }
$$

Of course, the tail of this vector is the position of $\underline{H}$ and the head $\underline{S}$. The time derivative of Eq. (5.1) is :

$$
\underline{\dot{v}}(t)=\mathbf{i} \Omega \cdot \underline{v}_{0} \mathrm{e}^{\mathbf{i} \Omega t}=\mathbf{i} \Omega \underline{v}(t) \quad(\text { or } \Delta \underline{v}=\mathbf{i} \Omega \Delta t \cdot \underline{v}) .
$$

Since $\underline{v}$ is equivalent to $\underline{H}_{\mathrm{r}}$, the right side of this equation corresponds to a horizontal torque. However, as nutation is free motion, no torque acts on the body. It is reasonable to consider the right side as an internal torque that changes not the tail but the head or direction of vector $\underline{v}$. The tail changes only after the external torque acts on the body.

Equation (3.7) is substituted into (5.2) to get

$$
\underline{\ddot{\theta}}-\mathbf{i} \Omega \underline{\dot{\theta}}=0 \text {. }
$$

This is equivalent to Eq. (4.6) when there is no external torque term.

\section{Eigenvalues and Root-Locus Method of the Linearized Equation.}

Substituting $\underline{\theta}=\mathrm{e}^{\lambda t}$ into Eq. (4.6), the following characteristic equation of $\lambda$ is obtained:

$$
\lambda^{2}+\left(k_{N}-\mathbf{i} \Omega\right) \lambda-\left(k_{g}+\mathbf{i} \Omega k_{P}\right)=0 .
$$

This equation is only quadratic. At high speed $\left(\Omega^{2}>>k_{g}, \Omega k_{P}\right)$, approximate values for the two eigenvalues $\lambda_{N}$ and $\lambda_{P}$ can be obtained by applying Taylor's expansion method to the square root term as follows:

$$
\begin{array}{lc}
\lambda_{N} \approx-k_{N}+k_{P}+\mathbf{i}\left\{\Omega-\left(k_{g} / \Omega\right)\right\} & \text { (fast or nutation mode or N-mode) } \\
\lambda_{P} \approx-k_{P}+\mathbf{i}\left(k_{g} / \Omega\right) & \text { (slow or precession mode or P-mode) }
\end{array}
$$

As long as $k_{P}>0$, or $r>0$, the P-mode is always stable. However, the stability condition of the $\mathrm{N}$-mode is a little complex; that is,

$k_{N}>k_{P}$ or $\sigma \equiv C / A>r / h \quad$ (stability condition for N-mode).

The N-mode of a tippe or mushroom-shaped top is unstable because $r / h>1$ and $\sigma \approx 1$ or 
$C \approx A$. This unstable condition for the tippe top agrees with the results obtained using a different method. ${ }^{(2)}$

The root-locus method can then be applied to various axisymmetric spinning bodies. For the varying parameter, $\Omega$ in the characteristic equation was selected. The loci of the characteristic roots are plotted on the complex plane as $\Omega$ increases from 0 . It should be noted that as the characteristic equation (6.1) has a complex coefficient and complex constant, the roots are not always conjugate; in other words, the loci are not always symmetric to the real axis. The method was applied under the condition that all rotating bodies are almost standing or that their polar axes are nearly vertical.

Figure 3 shows six root-loci where Cases (1), (4), and (6) are frictionless; the other three Cases (2), (3), and (5) are examples with friction. The three half circles of Cases (1), (2) and (3) are very helpful for understanding the shapes of each locus where $k_{\mathrm{g}}>0$. In particular, Case (1) is a fundamental locus so that when two roots meet on the way, it becomes a double-root; both separate up and down thereafter. We can frequently observe large abrupt coning motions when the ordinary spinning top decreases $\Omega$ and approaches the double-root point. This is a beat phenomenon, i.e., the two mode frequencies approach mutually. Case (6) is a very special case where no external torque acts and the starting point is the origin-i.e. the half-circle degenerates to double roots. In this case, P-mode is always zero, and the coning motion is called nutation in space engineering.

Nutation of the satellite is a coning motion around a fixed angular momentum vector on which no external torque acts; it corresponds to the zero root. The spinning boiled egg of Case (3) has a far larger $r$ compared to Case (2); therefore, the stability margin of the $\mathrm{N}$-mode at high speed is very small compared to the ordinary top of Case (2). It is clear that the tippe top at high spinning speed has an unstable N-mode and begins to enlarge its cone angle at the start of spin. For roundish spinners, generally the stability margin of the $\mathrm{N}$-mode is small or negative, although the stability margin of P-mode is very large.

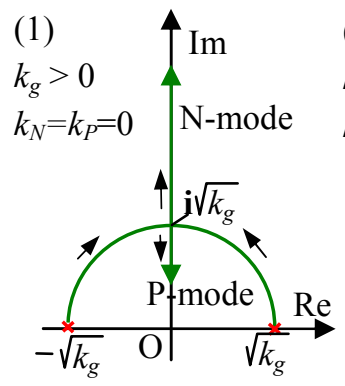

frictionless spinning top
(2)

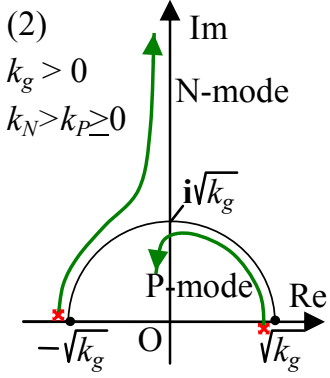

spinning top

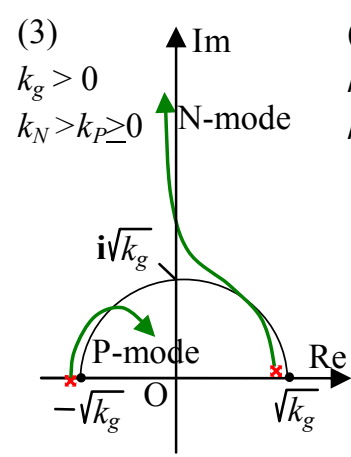

spinning boiled egg
(4)

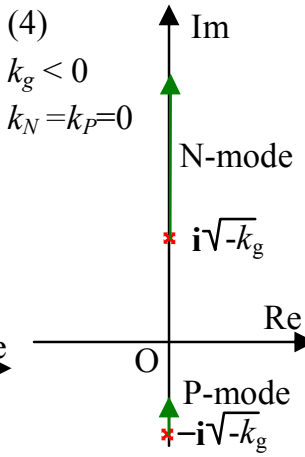

frictionless tippe top*, discus

Characteristic Equation

$\lambda^{2}+\left(k_{N}-\mathbf{i} \Omega\right) \lambda-\left(k_{g}+\mathbf{i} \Omega k_{P}\right)=0$

( $\Omega$ : varying parameter)

x : starting point $(\Omega=0)$

tippe top*

$=$ mushroom-shaped top

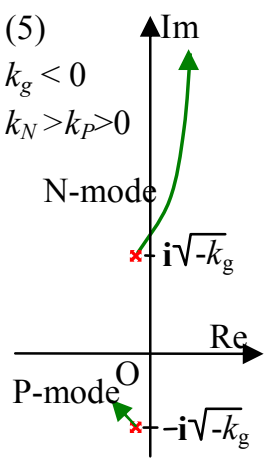

tippe top*, discus

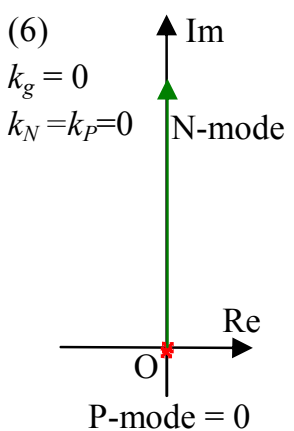

spinning satellite

Fig. 3 Root Locus Method Applied to Various Spinning Bodies 
As the discus of Case (5) has a larger $k_{\mathrm{p}}$ than the tippe top, the speed of divergence for the $\mathrm{N}$-mode is faster. The inclination angle $v$ increases up to $\pi / 2$ which is the limit for the unstable region because of shape symmetry, the body spins stably on its edge (not shown here).

\section{State Equation and Expression Using Transition Matrix.}

First, the derivation of a state equation from Eq. (4.6) is attempted under the precondition that the two eigenvalues $\lambda_{N}$ and $\lambda_{P}$ are given. Using complex variables, the state variables are expressed as follows:

$$
\underline{\theta}_{1} \equiv \underline{\theta}, \quad \underline{\theta}_{2} \equiv \underline{\dot{\theta}}_{1}, \quad \boldsymbol{x} \equiv\left[\underline{\theta}_{1} \underline{\theta}_{2}\right]^{\mathrm{T}} .
$$

Applying the relation between roots and coefficients of the characteristic Eq. (6.1), Eq. (4.6) can be expressed as

$$
\underline{\dot{\theta}}_{2}=\left(\mathbf{i} \Omega-k_{N}\right) \underline{\theta}_{2}+\left(k_{g}+\mathbf{i} \Omega k_{P}\right) \underline{\theta}_{1}=\left(\lambda_{N}+\lambda_{P}\right) \underline{\theta}_{2}-\lambda_{N} \lambda_{P} \underline{\theta}_{1}
$$

The following simple coefficient matrix $\boldsymbol{A}$ of the typical state equation $\dot{\boldsymbol{x}}=\boldsymbol{A x}$ is obtained:

$$
\boldsymbol{A} \equiv\left[\begin{array}{cc}
0 & 1 \\
-\lambda_{N} \lambda_{P} & \lambda_{N}+\lambda_{P}
\end{array}\right]
$$

The output of the state equation $\boldsymbol{y}$ is selected here as follows:

$$
\begin{aligned}
\boldsymbol{y} & \equiv\left[\begin{array}{llll}
\underline{S} & \underline{P} & \underline{\omega} & \underline{H}
\end{array}\right]^{\mathrm{T}}=\boldsymbol{C} \boldsymbol{x} \\
\boldsymbol{C} & =\left[\begin{array}{cccc}
-\mathbf{i} & \mathbf{i}(1-(r / h)) & -\mathbf{i} & -\mathbf{i} \\
0 & 0 & \sigma / \Omega & 1 / \Omega
\end{array}\right]^{\mathrm{T}} .
\end{aligned}
$$

The state variable $\boldsymbol{x}(t)$ is easily obtained from its initial condition $\boldsymbol{x}(0)$ by multiplying the transition matrix $\Phi(t)$ from the left side. The following Eq. (7.6) is very effective for expressing animation by selecting proper time intervals.

$$
\boldsymbol{x}(t)=\boldsymbol{\Phi}(t) \boldsymbol{x}(0) \text {. }
$$

$\Phi(t)$ is obtained through inverse Laplace transformation of $(s \boldsymbol{I}-\boldsymbol{A})^{-1}$, where $s$ is the Laplace transform operator, and $\boldsymbol{I}$ is the unit matrix. Here, only the results are shown.

$$
\boldsymbol{\Phi}(t)=\frac{1}{\lambda_{N}-\lambda_{P}}\left[\begin{array}{cc}
\lambda_{N} \mathrm{e}^{\lambda_{P} t}-\lambda_{P} \mathrm{e}^{\lambda_{N} t} & \mathrm{e}^{\lambda_{N} t}-\mathrm{e}^{\lambda_{P} t} \\
-\lambda_{N} \lambda_{P}\left(\mathrm{e}^{\lambda_{N} t}-\mathrm{e}^{\lambda_{P} t}\right) & \lambda_{N} \mathrm{e}^{\lambda_{N} t}-\lambda_{P} \mathrm{e}^{\lambda_{P} t}
\end{array}\right] .
$$

Figure 4 shows an example of an ordinary spinning top calculated using this method, where eleven $\underline{v}$ vectors are added at only the beginning part; these are shown by green arrows. The N-mode decays relatively quickly, while the P-mode decays slowly. The black arrow A shows a cusp on which the main three attitude vectors, $\underline{S}, \underline{H}$, and $\underline{\omega}$ coincide. In the following section, the locus of $\underline{S}$ near this cusp is shown to be an approximate cycloid.

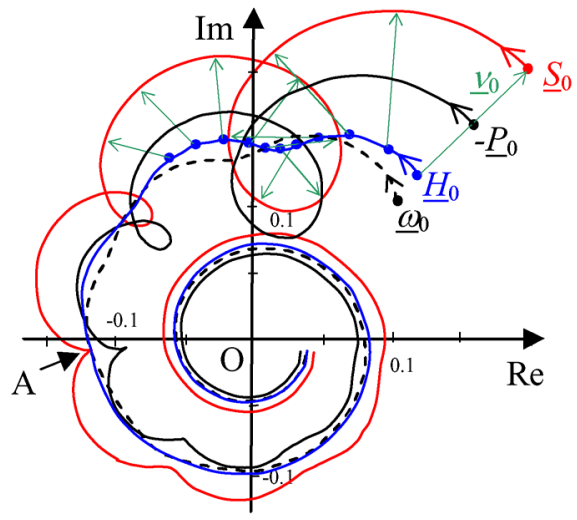

Simulation data:

$\lambda_{\mathrm{N}}=-4.3145614890245$

$+44.2884067096 * \mathbf{i}$

$\lambda_{\mathrm{P}}=-0.68543851097549$

$+5.711593290334 * \mathbf{i}$

$\Omega=50$

$k_{\mathrm{N}}=5, \quad k_{\mathrm{P}}=1.1, \quad k_{g}=250$

$\underline{\theta}_{0}=0.2-0.2 * \mathbf{i}$

$\dot{\theta}_{0}=4+4 * \mathbf{i}$

$t=0 \sim 2$

Fig. 4 Example of Application Using Transition Matrix.

\section{Analytical Solution of a Horizontally Suspended Spinning Top.}

When a horizontally spinning top is suspended by bearing systems or a string at a point 
located at some distance from the center of gravity, it is acted on by a nearly constant gravity torque $\boldsymbol{Q}_{\mathrm{g}}$ as long as the attitude does not significantly leave the horizontal plane. If the suspension system has no friction, then the only external torque is the constant gravitational torque and we can get an analytical solution. Moreover, a very simple physical model can be obtained.

In this case, the coordinate system should be changed: the complex coordinate plane is not horizontal but vertical, where the Re- or $X$-axis is part of the equator of the inertial unit sphere, the origin $O$ is on the sphere near $S$, and the Im- or $Y$-axis is part of a meridian passing through the origin, as shown in Fig.5. In this case, $\boldsymbol{Q}_{\mathrm{g}}$ becomes the real number $Q_{\mathrm{g}}$.

The equation of motion then becomes a very simple form:

$$
\underline{\ddot{\theta}}-\mathbf{i} \Omega \underline{\dot{\theta}}=Q_{g} / A \equiv q_{g} .
$$

This equation is easy to solve through Laplace transformation. The result is

$$
\begin{aligned}
\underline{S}(t) & \equiv-\mathbf{i} \underline{\theta}(t)=\underline{S}_{0}+\left(q_{g} / \Omega^{2}\right) \Omega t-\left(\underline{v}_{0}+\mathbf{i}\left(q_{g} / \Omega^{2}\right)\right)\left(1-\mathrm{e}^{\mathrm{i} \Omega t}\right) \\
& =\underline{H}_{0}+\left(q_{g} / \Omega^{2}\right) \Omega t-\mathbf{i}\left(q_{g} / \Omega^{2}\right)+\left(\underline{v}_{0}+\mathbf{i}\left(q_{g} / \Omega^{2}\right)\right) \mathrm{e}^{\mathbf{i} \Omega t} .
\end{aligned}
$$

From this solution, it is easy to show that the loci of $\underline{S}$ are trochoid curves using a rolling wheel on a straight rail model, as shown in Fig. 5. In Fig. 5, four loci of $\underline{S}(\mathrm{t})$ with different $\underline{S}_{0}$ or $\underline{v}_{0}$ are shown. $\underline{S}_{0}$ is on the edge of a flange whose radius may be larger or smaller than the radius of the rolling wheel depending on $\underline{v}_{0}$. The contact point of the wheel to the rail is $\underline{H}(\mathrm{t})$, which moves towards the right side with the constant velocity from the second term of Eq. (8.2). If the radius of the flange is larger than the wheel, then the locus includes loop parts as shown by the red curve; when it is smaller, the loci do not include the loop. If $\underline{S}_{0}$ is on the edge of the rolling wheel, then the locus becomes a cycloid. Another special case is where $\underline{S}_{0}$ is on the center of the wheel; the locus is a straight line which does not include the N-mode. Although the N-mode decays away, the nutation action continues. $\underline{S}$ rotates around $\underline{H}$; however, the speeds of both $\underline{H}$ and $\underline{S}$ are identical, so their loci become two parallel straight lines. If only a small $K_{N}$ term is included, then $\underline{S}$ pulls $\underline{H}$ down a little. This effect is shown in the next section using Fig. 6.

If, $k_{\mathrm{g}}$ or $q_{\mathrm{g}}<0$ such as in Cases (4) and (5) in Fig.3, then the rolling wheel is in the upper side of the rail and goes toward the left side, and $\underline{S}$ without the loop is always on the upper side of $\underline{H}$. If only a small $K_{N}$ term is included again, then $\underline{S}$ draws $\underline{H}$ up this time, though by a very small amount. The two parallel lines become two retrograde precession curves that approach the zenith very slowly. This effect is shown in Section 12 using Eq. (12.1).

It is intuitively understood that each time, $\underline{S}$ rotates around the instantaneous $\underline{H}$, which is the contact point of the rolling wheel to the rail.

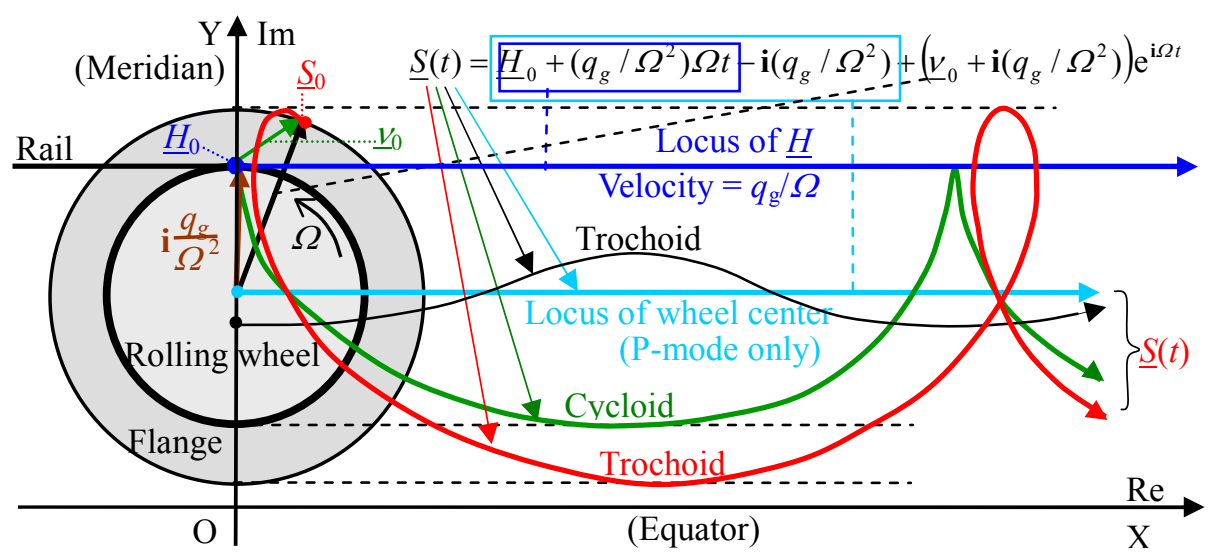

Fig. 5 Physical model of behavior for a horizontally suspended spinning top. 


\section{Discussion about Behavior of $H$ and $S$ Using Figs.4 and 5.}

It is not so difficult to obtain an analytical solution without using eigenvalues for Case (1) in Fig. 3, which is an ordinary vertical spinning top without friction under the condition that the amplitude of high speed mode is relatively small so that the gravitational torque can be considered as constant. In this case, the locus of $\underline{S}$ becomes an epitrochoid that includes an epicycloid, although it is not shown in this paper. The physical model is very similar to Fig. 5. The main difference is the shape of the rail: the straight line in Fig. 5 becomes a circle whose center is the origin of the complex coordinate plane. This similarity is very convenient for discussing the behavior of ordinary spinning tops. For example, the locus near the cusp in Fig. 4 is nearly an epicycloid, which corresponds to the cycloid in Fig.5.

Figure 6 shows an approximate epitrochoid in (a) and an approximate epicycloid in (b); these are cases of Fig. 4 without $\boldsymbol{Q}_{\mathrm{f}}$ and correspond to the trochoid and cycloid in Fig. 5, respectively. The locus of $\underline{H}$ in (a) is not a simple circular arc but includes the $\mathrm{N}$-mode effect; this is because $k_{g} \underline{\theta}$ is no longer constant unlike the case for Fig. 5. This effect is shown by two arrows $\underline{H}_{1}$ and $\underline{S}_{1}$ at the same time. Their phase angles are quite different. In Fig. 4, we can see that the directions of $\underline{v}=\underline{S}-\underline{H}$ in the first loop change considerably. The $\boldsymbol{Q}_{\mathrm{g}}$ is perpendicular to $\underline{S}_{1}$, and this direction coincides with the tangential line of the curve of $\underline{H}$ at $\underline{H}_{1}$. Figure 6(b) shows the effect of the $k_{N}$ term. In (b), the curve of $\underline{H}$ becomes nearly a circle, though not exact, and swells outwards as it approaches the $\underline{S}$ curve. This move by $\underline{H}$ corresponds to the downwards movement of the rail in Fig. 5. The approach of $\underline{H}$ to $\underline{S}$ also means that $\underline{S}_{0}$ approaches the center of the rolling wheel in Fig. 5; finally, the locus of $\underline{S}$ becomes the straight line indicated in light blue.

Through the weak $K_{p}$ effect in Fig.4, $\underline{H}$ gradually moves towards the origin and $q_{g}$ becomes smaller; therefore, the radius of the rolling wheel becomes smaller. As a result, the parallel spirals of $\underline{S}$ and $\underline{H}$ approach each other, and their moving velocities decrease.

In the first loop of $\underline{S}$ in Fig. 4, the approach of $\underline{H}$ to $\underline{S}$ is due to not only $k_{N}$ but also $k_{P}$. The velocity variation of $\underline{H}$ is recognized by observing the distribution of the small blue circles marked at identical intervals of time on the locus of $\underline{H}$ in Fig. 4. The large inclination of $\underline{S}$ introduces a large moving wheel and fast moving velocity, and vice versa.

(a)

$\approx$ Epi-trochoid (includes large $\mathrm{N}$-mode)

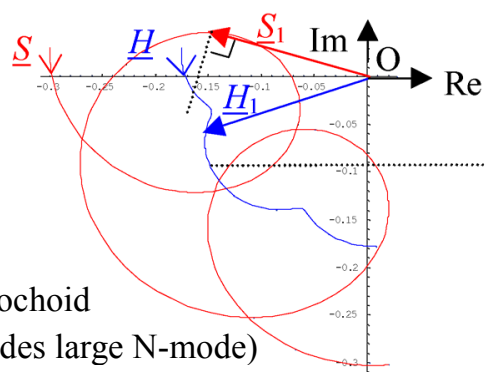

(b)

$$
\begin{aligned}
& \approx \text { Epi-cycloid } \\
& \quad\left(\text { after } k_{\mathrm{N}} \text { decay }\right)
\end{aligned}
$$

Fig. 6 Loci of $\underline{H}$ and $\underline{S}$ with variable $q_{\mathrm{g}}$ and zero $q_{\mathrm{f}}$ of Fig.4.

\section{Mechanism of Behavior of N-mode}

It is important to note that under the external torques, the direction of the spin axis $\boldsymbol{S}$ does not abruptly change its direction, but both the center of the cone or direction of $\boldsymbol{H}$ and the magnitude of $\boldsymbol{H}$ or coning velocity $\Omega$ are changed even though the external torque is impulsive. The real part of $\lambda_{N}$ (Eq. (6.2)) brings $\underline{H}$ close to $\underline{S}$ or keeps $\underline{H}$ away from $\underline{S}$, or in other words, the sign of the real part decides convergence or divergence of the N-mode.

In Eq. (4.1), the two external torques $\boldsymbol{Q}_{f}$ and $\boldsymbol{Q}_{g}$ are supposed to be small; therefore, the approximate superposition principle can be applied. Using only $\boldsymbol{Q}_{f}$ is sufficient to check the 
stability of N-mode because only the real part of the eigenvalue, which does not include $k_{g}$, is necessary. This is looked into in more detail.

First, the spin axis $\boldsymbol{S}$ is assumed to draw a cone whose center axis is $\boldsymbol{H}$, and the half cone angle is $v$ under the condition of weightlessness and no friction, such as a spinning satellite with a nutation motion whose velocity is $\Omega$ (Case(6) of Fig.3). The initial $\boldsymbol{H}$ or $\boldsymbol{H}_{0}$ is assumed to be sufficiently large, and its direction coincides with the $Z$ axis. Next, the state where both the friction and gravity torques have small and constant magnitudes is considered to act suddenly to $\boldsymbol{H}$. Assume $\underline{H}_{0}=0$ and that $\underline{S}_{0}$ is a little away on the real axis from the origin. Accordingly, the initial direction of $\boldsymbol{Q}_{f}$ is also the positive direction of the real axis provided that $h>>r$; or, the axis of the top is assumed to be very thin and $k_{N}>>k_{P}$ $\approx 0$.

Similarly, the initial direction of $\boldsymbol{Q}_{g}$ is the positive direction of the imaginary axis from the origin.

The two torques have constant magnitude as mentioned before; however, they change their directions with the circulating velocity of the contact point $P$, which is nearly equal to $\Omega$. Thus, we can express the torques in the following approximate equation whose forcing term is a superposed function of $Q_{f}$ and $Q_{g}$ :

$$
\underline{\ddot{\theta}}-\mathrm{i} \Omega \underline{\dot{\theta}}=\left(\underline{Q}_{f}+\underline{Q}_{g}\right) / A \approx\left(q_{f}+\mathrm{i} q_{g}\right) \mathrm{e}^{\mathrm{i} \Omega t},
$$

where both $q_{f}$ and $q_{g}$ are constant real numbers.

Equation (10.1) can be solved easily as in Section 8,

$$
\begin{aligned}
\underline{S}(t) & =\underline{S}_{0}+\left(\underline{\theta}_{0} / \Omega\right)\left(1-\mathrm{e}^{\mathrm{i} \Omega t}\right)+\left\{\left(\mathrm{i} q_{f}-q_{g}\right) / \Omega^{2}\right\}\left[1-(1-\mathrm{i} \Omega t) \mathrm{e}^{\mathrm{i} \Omega t}\right] \\
& =\underline{H}_{0}+\underline{v}_{0} \mathrm{e}^{\mathrm{i} \Omega t}+\left\{\left(\mathrm{i} q_{f}-q_{g}\right) / \Omega^{2}\right\}\left[\left(1-\mathrm{e}^{\mathrm{i} \Omega t}\right)+\mathrm{i} \Omega t \mathrm{e}^{\mathrm{i} \Omega t}\right] .
\end{aligned}
$$

The first and second terms on the right of Eq. (10.2) denote a free circular motion whose center is $\underline{H}_{0}(=0)$ and radius is $\underline{v}_{0}$ with a rotating velocity of $\Omega$. The third term on the right of Eq. (10.2) consists of $q_{f}$ and $q_{g}$ terms independent of each other; their effects are explained in Fig. 7. The coefficient of the square brackets corresponds to the negative radius of each circle as shown by black arrows.

In Fig. 7, (a) and (b) explain the effects of $q_{f}$ and $q_{g}$, respectively. The first term in the square brackets denotes the positions on each circle of $\underline{H}$ drawn by the external torques shown as $\underline{H}_{f}$ or $\underline{H}_{\mathrm{g}}$. The second term denotes the arc length of each circle, with phase angle change. Of course, if their signs change, the positions of their circles change symmetrically with respect to the Re- or Im- axis, respectively. For explanation of the $\mathrm{N}$-mode stability, (a) is important. In this case $q_{g}=0$; omitting $\underline{H}_{0}(=0)$, Eq. (10.2) becomes

$$
\begin{aligned}
\underline{S}(t) & =\mathrm{i}\left(q_{f} / \Omega^{2}\right)\left(1-\mathrm{e}^{\mathrm{i} \Omega t}\right)+\left[\underline{v}_{0}-\left(q_{f} / \Omega^{2}\right) \Omega t\right] \mathrm{e}^{\mathrm{i} \Omega t} \\
& =\underline{H}_{f}(t)+|\underline{v}(t)| \mathrm{e}^{\mathrm{i} \Omega t}=\underline{H}_{f}(t)+\underline{v}(t),
\end{aligned}
$$

where $|\underline{v}(t)|=\left[\underline{v}_{0}-\left(q_{f} / \Omega^{2}\right) \Omega t\right]$, in which for the length $\underline{v}(t)$, the decreased length is the arc length of the $q_{f}$ circle. The decreased amount is the amount by which $\underline{H}$ approaches $\underline{S} . \underline{v}$ ( = $\underline{S}-\underline{H}$ ) takes the role of the thread wound on the circle; as a result, $\underline{S}$ draws an involute curve. Thus, the eigenvalue of the $\mathrm{N}$-mode can be understood intuitively through these
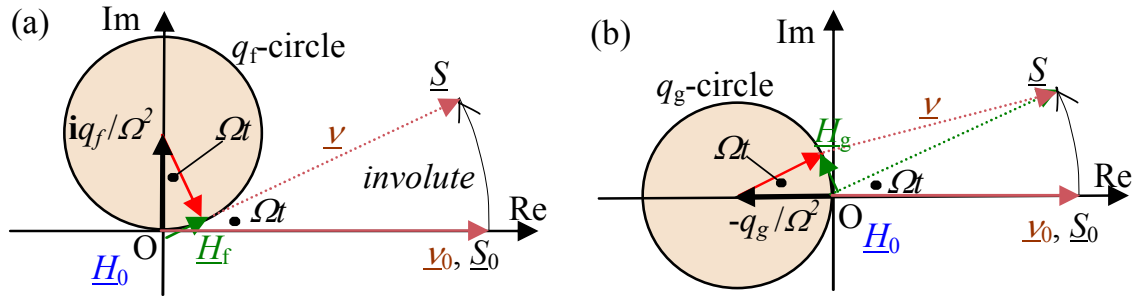

Fig. 7 Effects of both $q_{\mathrm{f}}$ and $q_{\mathrm{g}}$ (green arrows) on $\underline{\nu}$-vectors. 
explanations.

On the other hand, for (b) Eq. (10.3) can be used again, provided that $q_{f}$ is exchanged to $\mathrm{i} q_{f}$. Therefore, the $q_{g}$-circle and $\underline{H}_{g}$ have an advantage of $\pi / 2$ compared to the $q_{f}$ circle and $\underline{H}_{f}$. The motions of $\underline{H}$ and $\underline{S}$ are almost parallel to each other, so $|\underline{y}|$ is almost unchanged; however, the phase angle of $\underline{v}(\equiv \angle \underline{v})$ changes, which means a change of the rotating velocity of $\underline{S}(t)$ around $\underline{H}(t)$.

For very short time intervals, $-k_{N} \underline{\dot{\theta}}$ can be used instead of $q_{\mathrm{f}} \mathrm{e}^{\mathrm{i} \Omega t}$ because the change of $|\underline{\dot{\theta}}|$ is small. Therefore, in very short time intervals, the $v$ thread can be thought of as drawing an involute curve around a $q_{f}$ circle drawn by the $-k_{N} \underline{\dot{\theta}}$ torque; the radius is not strictly constant, but the change is negligible. In other words, the radius of the $q_{f}$ circle changes gradually, and the circle is always tangential to the $\underline{v}$ vector. As a result, $\underline{S}$ steadily draws an approximate involute curve. An example of this $q_{f}$ circle is shown in Fig. 8 in the next section.

\section{Expression for Large Inclination Angles}

As shown in Fig. 1, each attitude vector starts from the center of gravity $G$ and penetrates through the unit sphere. Even if each penetration point on the sphere is far from the zenith unlike in Fig.1, the position of each can be expressed in the complex coordinate plane through the azimuthal equidistant projection method frequently used in the map. The increase in $\underline{v}$ is a result of time integration of $-\underline{H}_{\mathrm{r}} / H_{\mathrm{p}}$, which is generated by only the horizontal component of the external torques. Therefore, the integrated $\underline{v}$ is equivalent to the arch length on the unit sphere. However, a large inclination introduces an increase in $q_{\mathrm{z}}$ or decrease in $\Omega$. If the initial value of $\Omega$ is large enough, then $\Omega$ can be considered as nearly constant for short time intervals. This method is very convenient when the inclination angle of the spinning axis is very large and a large $\boldsymbol{H}$ is near the $Z$-axis. Therefore, to express the locus of $\underline{S}$ on the map as the solution when using the fundamental Eq. (4.6), there is the following condition:

" $\underline{H}$ should be near the origin, and either $\Omega$ should be large enough or integration time should be short."

Here, $\underline{\dot{\theta}}(0)$ is given as $\dot{\theta}(0) \equiv-\Omega \underline{\underline{u}}(0)$ using Eq. (3.7) to solve the fundamental Eq. (4.6). $\underline{v}(0)$ is a complex number vector whose length is the arc length of $v(0)$; it starts from $\underline{H}$ and ends at $\underline{S}$ on the map.

Figure 8 shows an example for the locus of $\underline{S}$ when the inclination angle $\underline{v}$ for a tippe top exceeds the horizon or equator. The locus of $\underline{H}$ is a small circle, as shown in "Enlarged $\underline{H}$," and is generated by a resultant vector of $q_{\mathrm{f}}$ and $q_{\mathrm{g}}$. The radius of the $q_{\mathrm{f}}$ circle in Fig. 7 is 0.012 , and its circumference is 0.075 ; this is nearly the same as the increase in $|\underline{y}|$ per each cycle of $\underline{S}$. Therefore, the behavior of $\underline{S}$ seems as if it is connected to the $q_{\mathrm{f}}$ circle by a thread wound on the circle; the thread unwinds as $\underline{S}$ rotates around it. As a result, the locus of $\underline{S}$ becomes a spiral rather than an involute curve because the central circle is very small and the thread is very long. For the phase angle of the right term for the simulation model shown in Fig. 8, the phase angle of $-\mathbf{i} \underline{\theta}(=\underline{S})$ rather than $\Omega t$ was adopted.

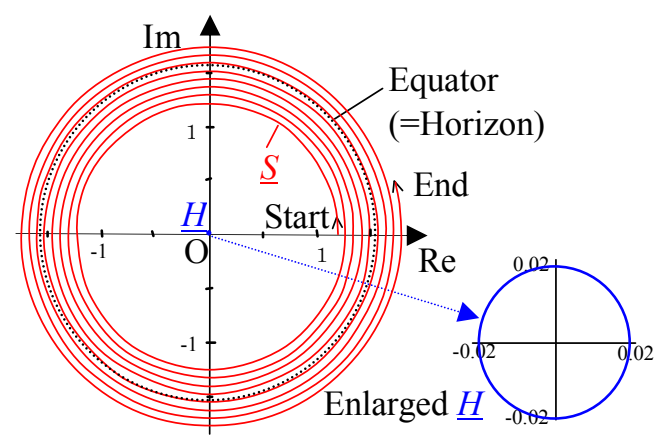

$$
\begin{aligned}
& \text { Simulation Model: } \\
& \begin{array}{l}
\ddot{\theta}-\mathrm{i} \Omega \underline{\dot{\theta}}=\left(q_{f}+\mathrm{i} q_{\mathrm{g}}\right) \mathrm{e}^{\mathrm{i} \angle(-\mathrm{i} \underline{\theta})} \\
\Omega=50, \quad q_{\mathrm{f}}=30, \quad q_{\mathrm{g}}=40 \\
\dot{\theta}(0)=-61, \underline{\theta}(0)=0+1.2 \mathrm{i} \\
t=0 \rightarrow 1.0 \\
\left|q_{\mathrm{f}}+\mathrm{i} q_{\mathrm{g}}\right| / \Omega^{2}=0.02 \\
q_{\mathrm{f}} / \Omega^{2}=0.012
\end{array}
\end{aligned}
$$

Fig. 8 Attitude locus of a tippe top beyond the horizon 


\section{Application to Control of Rotating Bodies}

The two modes of the fundamental equation (Eq. (4.5)) shown in Section 6 are stable if the coefficients $K_{N}, K_{P}$, and $K_{g}$ are properly selected.

Although the terms including these coefficients are the inevitable external torques, these terms can be considered as control torques when the spinning bodies are controlled, as in magnetic bearing systems. $K_{N}$ is a damping term because it decreases $\dot{\theta}$; in other words, it brings $\underline{H}$ close to $\underline{S}$. $K_{g}$ is rather familiar because it is known as the gravity stabilizing term. In magnetic bearing systems, usually $K_{g}<0$, which means the system is statically stable, such as for the tippe top. For high-speed rotation, however, this term has little effect on stabilization. It rotates $\underline{H}$ around the origin. When $K_{g}<0$, P-mode becomes a retrograde precession with weak stability even if $K_{P}=0$. This weak stability comes from the second-order term in Taylor's expansion series, which is neglected in Eq.(6.3):

$$
\lambda_{P} \approx-k_{P}+\left(k_{N} k_{g} / \Omega^{2}\right)+\mathbf{i}\left(k_{g} / \Omega\right) .
$$

The $K_{P}$-term can be rewritten as:

$$
K_{P} \text {-term }=\mathbf{i} \Omega K_{P} \underline{\theta}=-\Omega K_{P} \underline{S} .
$$

Clearly, the action of this term is to bring $\underline{H}$ to the origin provided that $\underline{S}$ is close to $\underline{H}$ or that stability of N-mode is adequate. Section 8 explains that when $K_{g}<0$, both $\underline{S}$ and $\underline{H}$ draw stable retrograde P-mode curves using only the $K_{N}$ term. However, this stability is very weak. Therefore, the $K_{P}$ term or the following cross-feedback method should be used.

Equation (4.5) includes two terms which are multiplied by $\mathbf{i}$. It is very easy to understand that these are the so-called cross-coupling terms. The first term $\mathbf{i} A \Omega \dot{\dot{\theta}}$ is known as the gyroscopic cross-coupling term. The second, the $K_{P}$-term, is also a cross-coupling term. If the latter term is wanted as a control signal in the magnetic bearing system, the signal $\underline{\theta}$ of the sensor is sent to the controller and multiplied by a proper gain; these two channel signals should then be cross-fed back to each actuator. It is interesting to note that the $K_{P}$-term is indispensable to an optimal regulator system for magnetic bearing systems ${ }^{(3)}$ and that the spinning top with a round shape for the contact point has this term naturally.

\section{Concluding Remarks}

A simple linearized equation of motion for spinning tops was established using the complex variables for attitude vectors and a concise expression of the friction torque. As a result, various convenient tools of linear theory could be used. The main tools used for this paper and their results are as follows:

1. Eigenvalues showing concrete geometric stability conditions.

2. The root-locus method for various axisymmetric rotating bodies to provide an intuitive understanding of attitude stabilities.

3. Derivation of simple expressions for the state equation and transition matrix.

4. From analytical solutions, a physical model explaining behavior of the top, and an intuitive explanation model of external torques to attitude stability are given.

5. Expression of large inclination angle using the azimuthal equidistant projection method.

6. Application for control of the magnetic bearing systems.

\section{References}

(1) Murakami, C., "All-Passive-Type Rotary Magnetic Bearing System Based on Stability Principle of Sleeping Tops", JSME International J. Series C, vol.38,No.3, 1995. pp.601-608.

(2) Sakai, T., "Sakadachi-goma", Suurikagaku211, (Jan. 1981), pp. 30-36, (in Japanese).

(3) Mizumo,T. and Higuchi,T., Design of the Control System of Totally Active Magnetic Bearings, Proc. of International Symposium on Design and Systems, (July, 1984), p.534-539. 